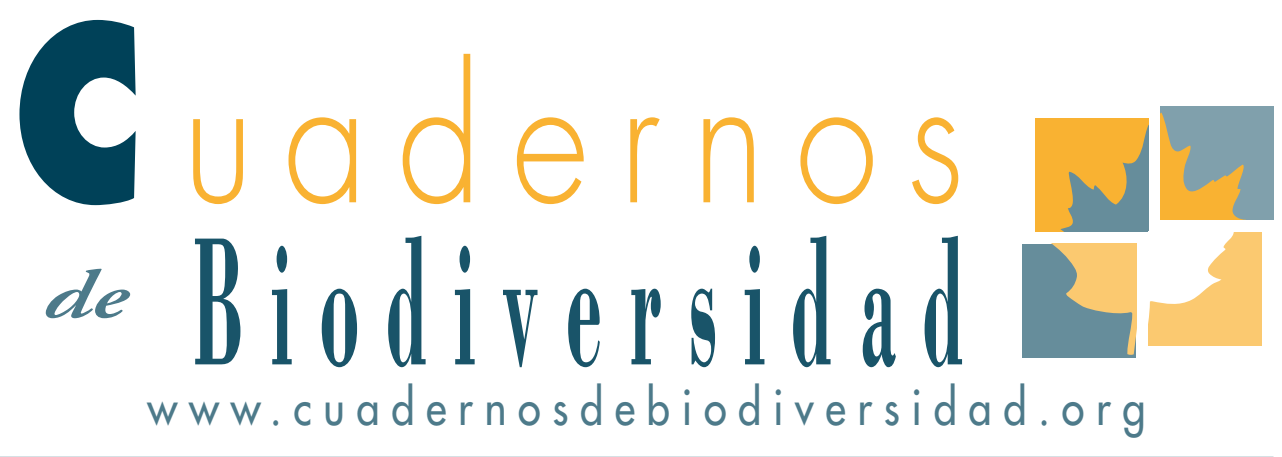

\title{
Influencia del recambio de especies en la biodiversidad de bosques tropicales: el ejemplo de Costa Rica
}

\section{A. García-López}

Centro Iberoamericano de la Biodiversidad CibiO, Universidad de Alicante, 03690 San Vicente del Raspeig, Alicante (España). Email: alejandra.garcia@ua.es

\section{ABSTRACT}

To understand the high heterogeneity of the biodiversity distribution is one of the most important challenges for the ecology. The study of this heterogeneity is not only ecologically interesting, but also urgent, because of the current biodiversity high degree of threat. Understanding the distributional patterns of species and the factors and mechanisms which are producing and maintaining these patterns is essential to consider protection and conservation strategies of the biodiversity in the most useful and effective way. The analysis of the distribution of scarab beetles assemblages (Coleoptera: Scarabaeidae: Dynastinae, Melolonthinae, Rutelinae) across the forests of Costa Rica revealed high species turnover increasing total diversity in these tropical ecosystems. Although environmental heterogeneity is responsible for part of this turnover, the historical and evolutionary factors have also an important role influencing species distribution. The incorporation of the analysis of these factors to the studies of diversity distribution represents a useful tool for the understanding of the processes producing the current biodiversity patterns.

\section{KEY WORDS:}

Diversity turnover, Dynastinae, Melolonthinae, Rutelinae, Scarabaeidae, species distribution. 


\section{INTRODUCCIÓN}

La heterogeneidad en la distribución de la biodiversidad es una de las más claras características del mundo natural que ya atrajo a los primeros naturalistas (Darwin, 1859; von Humboldt, 1849) y que hoy en día sigue constituyendo una de las principales preguntas en ecología. Conocer y entender los patrones de diversidad existentes y los mecanismos que los producen continúa siendo uno de los retos científicos más importantes (Gaston, 2000). La necesidad de documentar y entender esos patrones y mecanismos se hace urgente dado el grado de amenaza al que actualmente se enfrenta la biodiversidad debido a factores que provocan cambios en la estructura de las comunidades como son la destrucción y la fragmentación del hábitat o el cambio climático (Ewers \& Didham, 2006; Grimbacher et al., 2008; Root et al., 2003; Thomas et al., 2004). La falta de información reduce nuestra capacidad para predecir cómo las especies responderán en un futuro a estas alteraciones. Para poder valorar cuáles son los métodos que pueden resultar más efectivos para preservar, proteger y regenerar la biodiversidad, necesitamos una mejor comprensión de los mecanismos que la generan y mantienen. Aunque los esfuerzos científicos llevan décadas centrados en buscar la respuesta a estas preguntas, hoy en día no existe todavía una explicación general para la distribución de la biodiversidad, ni siquiera para los gradientes más ampliamente reconocidos y estudiados como son la latitud y la elevación (Brown, 2001; Lomolino, 2001; Rosenzweig, 1995). Esto pone de relieve la necesidad de una mayor información sobre los patrones geográficos de distribución de las especies en el mundo (Brown, 2001; Lomolino, 2001) y enfatiza el interés de estudios que nos ayuden a aumentar nuestro conocimiento sobre los factores y procesos relacionados con la producción y el mantenimiento de la biodiversidad.

Actualmente se ha demostrado la relación de numerosas variables climáticas y ecológicas presentes en los ecosistemas con la distribución de la diversidad. Factores como el clima (McCain, 2007; Ødegaard, 2006), la altitud (Kessler, 2009; Kumar et al., 2009), la latitud (Willig et al., 2003), factores físicos del terreno (Novotny \& Weiblen, 2005), el grado de degradación o modificación del hábitat (Nichols et al., 2007) y las dinámicas temporales (Escobar et al., 2008) entre otros, han demostrado en distintos casos su relación significativa con la diversidad. Sin embargo, el establecimiento y mantenimiento de las especies en las comunidades en las que las encontramos, también dependen de factores históricos y evolutivos (Hawkins et al., 2003; Ricklefs, 2004; Svenning et al., 2006) que influyen en la estructura de estas comunidades a lo largo de su historia. Comprender cómo intervienen estos factores es esencial para entender la biodiversidad y salvaguardar su futuro a través de la conservación de los procesos evolutivos tanto como de los patrones que estos han producido.

\section{BIODIVERSIDAD EN BOSQUES TROPI- CALES. EL CASO DE COSTA RICA}

Los bosques tropicales son los ecosistemas terrestres con mayor diversidad local de especies, con la estructura ecológica más compleja y mayor heterogeneidad espacial. La importancia de estos bosques radica en la variabilidad de hábitats que poseen (Myers et al., 2000), en su potencial biótico y genético, en su grado de endemicidad y en la presencia de paisajes únicos (Dinerstein et al., 1995), características que promueven la elevada biodiversidad que albergan. Ocupando solo un $7 \%$ de la superficie terrestre, los bosques tropicales contienen alrededor de la mitad de todas las especies presentes en el planeta (Thomas \& Baltzer, 2002).

Hoy en día la modificación, degradación y fragmentación de los bosques pone en peligro la integridad de estos ecosistemas en todo el mundo (Gascon et al., 2000; Harvey \& Sáenz, 2008). Esta alteración de los paisajes naturales por razones antropogénicas es la causa principal de la pérdida de biodiversidad actual (Foley et al., 2005; Reid et al., 2005). En el caso concreto de los bosques tropicales, el impacto de estos procesos de fragmentación, 
debido principalmente a la creciente deforestación resultado del desarrollo agrícola y ganadero, es el mayor responsable de la pérdida de diversidad biológica en el ámbito global (Kattan, 2002) (Fig. 1). No en vano, el manejo sostenible de los bosques tropicales fue identificado por la FAO (2005) como el principal objetivo para la conservación global de la biodiversidad puesto que son los biomas más diversos y amenazados del planeta.

Sin embargo, a pesar de su enorme interés ecológico y de la sustancial pérdida de superficie que sufren actualmente, el conocimiento de estos bosques es todavía mucho menor que el de otros tipos de ecosistemas (Longino, 1994), poniendo de relieve la importancia de estudios centrados en las áreas del planeta donde se ubican.

Los bosques de América Latina son los bosques tropicales más importantes del mundo, tanto por su extensión geográfica como por su riqueza biológica y complejidad ecológica (Hartshorn, 2002). Costa Rica forma parte del hotspot centroamericano (Myers et al., 2000) e incluye en su territorio parte de esos bosques. Con sólo $51.100 \mathrm{~km} 2$ de superficie terrestre (representando únicamente el 0,03\% de la mundial), Costa Rica es considerado uno de los 20 países con mayor biodiversidad, mostrando la densidad de especies (número de especies por unidad de área) más alta en el mundo (Obando, 2002; Valerio, 1999). Las especies que se encuentran en este pequeño territorio representan cerca del $4 \%$ del total de las especies estimadas a nivel mundial. Costa Rica alberga, además de esa elevada riqueza de especies, una enorme diversidad de ecosistemas que van desde paisajes litorales localizados a nivel del mar hasta paisajes de páramo sobre los 3.000 metros de altitud (Harsthorn, 1991).

Algunas de las razones que explican esa enorme riqueza natural del país, tanto en especies como en ecosistemas, son su posición geográfica en el Neotrópico, la presencia de dos vertientes costeras, su historia geológica como puente ístmico (Bergoeing, 1998), constituyendo una importante área de transición entre dos masas continentales (Valerio, 1999) y la existencia de un sistema montañoso que provee numerosos y variados microclimas y que dota al país de una topografía general muy característica. La Cordillera de Guanacaste $(112 \mathrm{~km})$, la Cordillera de Tilarán $(50 \mathrm{~km})$, la Cordillera Central $(76 \mathrm{~km})$ y la Cordillera de Talamanca $(180 \mathrm{~km})$ atraviesan el país de noroeste a sudeste abarcando prácticamente su extensión total (Fig. 2).

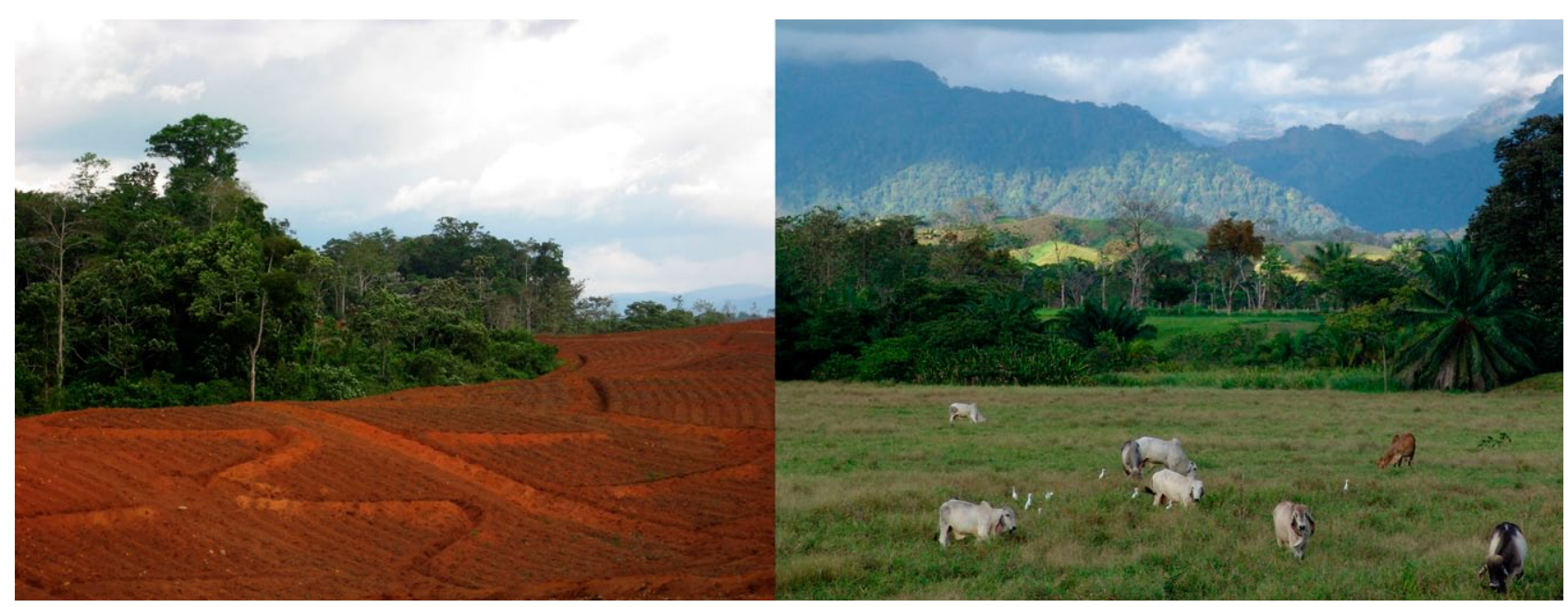

Fig. 1. Áreas deforestadas para uso agrícola y ganadero en Costa Rica. 


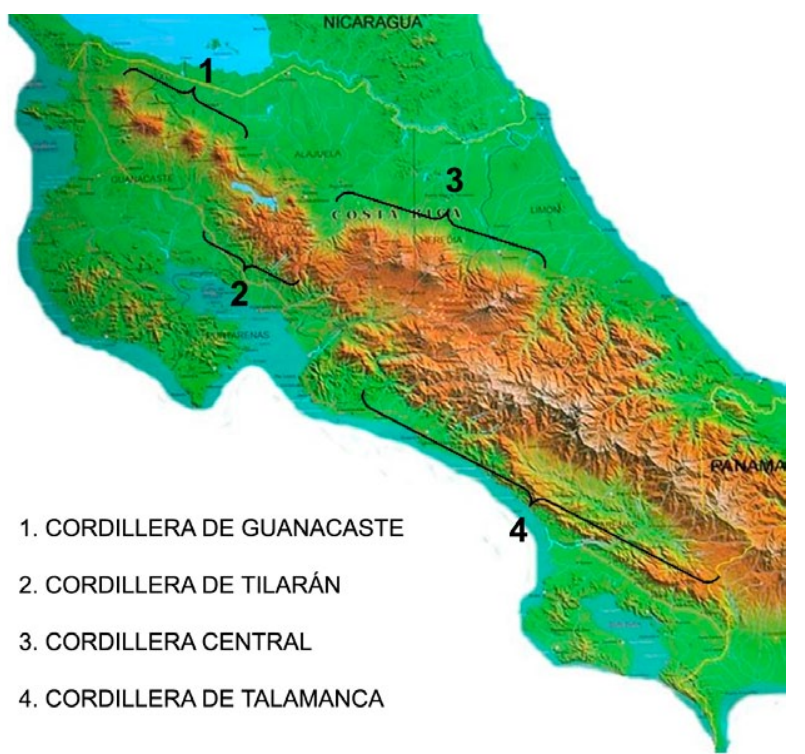

Fig. 2. Distribución de las áreas montañosas en Costa Rica.
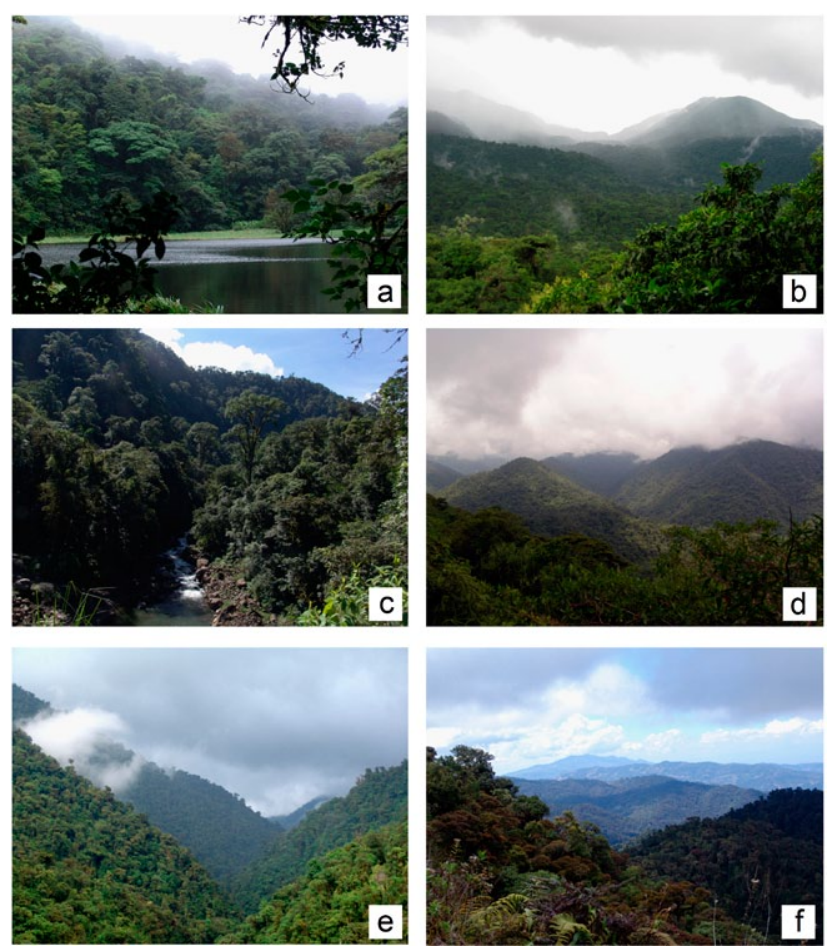

Fig. 3. (a) Laguna Danta. Cordillera de Guanacaste; (b) Parque Nacional Volcán Tenorio. C. de Guanacaste; (c) Parque Nacional Braulio Carrillo. C. Central; (d) Río Cariblanco. C. Central; (e) La Esperanza del Guarco. C. de Talamanca; (f) Parque Nacional Tapantí. C. de Talamanca

montañoso del país (dos puntos en la Cordillera de Guanacaste, dos puntos en la Cordillera Central y dos puntos en la Cordillera de Talamanca). Todos estos puntos de muestreo se situaron en una franja de altitud entre los 900 y 1100 metros de manera que todos se encontraran en la misma zona de vida, el bosque lluvioso premontano, y así mantuvieran una homogeneidad ambiental que permitiese evaluar la variación de diversidad cuando las características del ecosistema se mantienen constantes (García-López et al., 2010). En el caso del transecto altitudinal seis puntos de muestreo fueron ubicados en bosques situados a lo largo de un gradiente de $1400 \mathrm{~m}$ de elevación en la Cordillera de Guanacaste, abarcando desde los 100 a los 1510 m (García-López et al., 2011). 


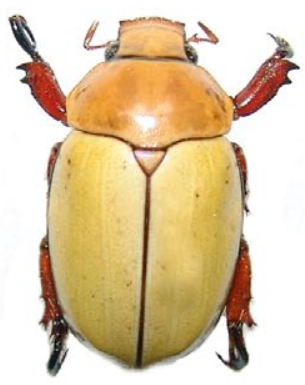

Phalangogonia sperata
(Sharp, 1878)
RUTELINAE

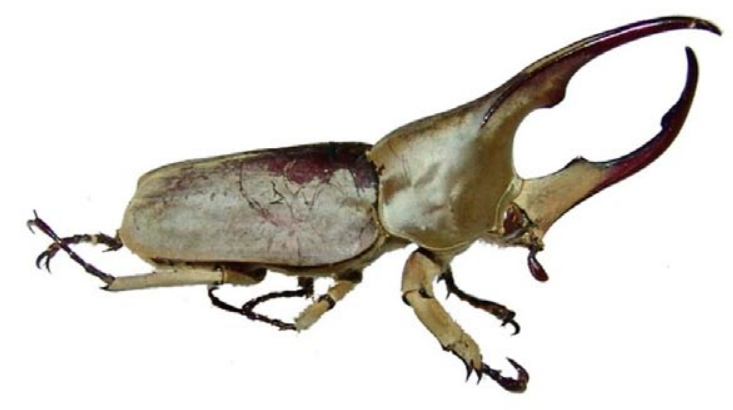

Spodistes beltianus
(Bates, 1988)
DYNASTINAE

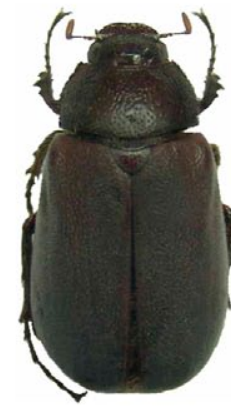

\section{Phyllophaga larai \\ (García-López \& Morón, 2009) MELOLONTHINAE}

Fig. 4. Subfamilias de Scarabaeidae pertenecientes al grupo de estudio.

Los resultados de estos estudios mostraron que la riqueza, abundancia y composición del grupo varían de manera significativa asociadas tanto al gradiente altitudinal, como dentro del mismo tipo de ecosistema en ausencia de grandes diferencias ecológicas. Esto pone de manifiesto el importante papel que el recambio de especies tiene en la diversidad total que encontramos en estos bosques. Pero, ¿qué factores influyen en ese elevado recambio de especies?

Espacialmente, la distribución de la diversidad a través de los bosques estudiados se encuentra influenciada por la topografía tan característica que define estos ecosistemas, distribuidos a lo largo de distintas montañas y cordilleras. En el caso de los sitios de muestreo dispuestos a través del bosque lluvioso premontano, existe un importante recambio de especies entre bosques que presenta gran influencia en la diversidad total. Teniendo en cuenta la similitud ambiental entre estos sitios de muestreo, tratándose en todos ellos de bosques semejantes en altitud, clima y estructura, esto indica que los procesos históricos, geográficos y evolutivos constituyen factores clave en la formación y mantenimiento de los ensambles de escarabeidos presentes en estos bosques. Los análisis de estructura filogenética de la comunidad llevados a cabo confirman esta influencia (García-López et al. En revisión). La elevada proporción de haplotipos y especies exclusivos de un solo sitio de muestreo y el alto recambio de diversidad (haplotípica, específica y filogenética) entre bosques, indican que el aislamiento asociado a procesos de alopatría entre montañas tiene un importante papel en determinar la distribución de la riqueza de especies de estos bosques en Costa Rica.

A pesar de la gran influencia del recambio de especies entre montańas en la diversidad total de los bosques de Costa Rica, los mayores cambios en la diversidad de los ensambles se encontraron asociados al gradiente altitudinal. Las diferencias más acusadas en la riqueza y composición de especies se encontraron entre los bosques de tierras altas (a más de $1000 \mathrm{~m}$ de altura) y los de tierras bajas (a menos de $1000 \mathrm{~m}$ ), donde el recambio de especies llegó a ser del 100\% en algunos casos. Los cambios ecológicos asociados a la altitud, como las variaciones en la estructura y composición vegetal del bosque ligadas a los gradientes de temperatura y humedad, son factores que influyen en la estructura de la comunidad. Siendo esas variaciones ecológicas mucho mayores en el gradiente altitudinal que en el caso del análisis a través del bosque lluvioso premontano, es esperable que los cambios en los ensambles asociados a ellas sean también más marcados. Sin embargo, a pesar de que la humedad y la temperatura condicionan aspectos tan importantes para las especies como su fisiología y la vegetación que forma parte de su nicho, es la elevación el factor que ha demostrado mayor influencia sobre la distribución de las especies. Dado que las tres variables se encuentran directamente relacionadas, encontrando a mayor elevación menor temperatura y mayor humedad y viceversa, la mayor influencia de la elevación frente 
a los factores ambientales que la definen, vuelve a señalar a los procesos evolutivos e históricos como factores clave en el establecimiento de los ensambles que actualmente encontramos.

\section{CONCLUSIÓN}

Los resultados indican que los bosques montanos de Costa Rica constituyen un importante centro de creación y mantenimiento de la biodiversidad. La gran heterogeneidad en la distribución de las especies, incluso dentro de un mismo tipo de ecosistema, hace necesarias estrategias de conservación que busquen abarcar esos gradientes, tratando en todo caso de asegurar la conexión entre los distintos bosques. La elevada concentración de diversidad a altitudes en las que la amenaza de la deforestación es mayor dada su accesibilidad y condiciones, que permiten mayor intensidad de procesos como el desarrollo urbanístico, la agricultura, ganadería y silvicultura, así como el efecto de aislamiento que esa deforestación produce y que podría llegar a ocasionar la pérdida irreversible de especies, hacen necesarias estrategias de control del grado de fragmentación y del proceso de reducción del hábitat en esas zonas.

La evidencia del papel fundamental que procesos históricos y evolutivos tienen sobre la forma en que las especies se distribuyen hoy en día hacen que trabajos que lleven a cabo análisis de riqueza y distribución de los ensambles de especies junto con el análisis de la diversidad y estructura filogenética de esos ensambles constituyan un puente entre la ecología y la evolución de gran utilidad como herramienta para la comprensión de los procesos que median en la coexistencia de las especies y, por lo tanto, en la conservación de la biodiversidad.

\section{REFERENCIAS}

BERGOEING, J.P. 1998. Geomorfología de Costa Rica. Instituto Geográfico Nacional. San José. Costa Rica. 409 pp.
BROWN, J.H. 2001. Mammals on mountainsides: elevational patterns of diversity. Global Ecol. Biogeogr., 10:101-109.

DARWIN, C. 1859. On the origin of species by means of natural selection or the preservation of favoured races in the struggle for life. John Murray. London. U.K.

DINERSTEIN, E., OLSON, D.M., GRAHAM, D.J., WEBSTER, A.L., PRIMM, S.A., BOOKBINDER, M.P. \& LEDEC, G. 1995. A conservation assesment of the terrestrial ecoregions of Latin America and the Caribbean. The World Wide Fund and World Bank, Washington, D.C. U.S.A. 129 pp.

ESCOBAR, F., HALFFTER, G., SOLÍS, Á., HALFFTER, V. \& NAVARRETE, D. 2008. Temporal shifts in dung beetle community structure within a protected area of tropical wet forest: a 35-year study and its implications for long-term conservation. J. Appl. Ecol., 45:1584-1592.

EWERS, R.M. \& DIDHAM, R.K. 2006. Confounding factors in the detection of species responses to habitat fragmentation. Biol. Rev., 81:117-146.

FAO. 2005. Global Forests Resources Assessment. URL: http://www.fao.org.

FOLEY, J.A., DEFRIES, R., ASNER, G.P., BARFORD, C., BONAN, G., CARPENTER, S.R., CHAPIN, F.S., COE, M.T., DAILY, G.C., GIBBS, H.K., HELKOWSKI, J.H., HOLLOWAY, T., HOWARD, E.A., KUCHARIK, C.J., MONFREDA, C., PATZ, J.A., PRENTICE, I.C., N., R. \& SNYDER, P.K. 2005. Global consequences of land use. Science, 309:570-574.

GARCÍA-LÓPEZ, A., MICÓ, E. \& GALANTE, E. 2011. From lowlands to highlands: searching for elevational patterns of species richness and distribution of scarab beetles in Costa Rica. Divers. Distrib., 18:543-553.

GARCÍA-LÓPEZ, A., MICÓ, E., NUMA, C. \& GALANTE, E. 2010. Spatiotemporal variation of scarab beetle assemblages (Coleoptera: Scarabaeidae: Dynastinae, Melolonthinae, Rutelinae) in the premontane rain forest in Costa Rica: a question of scale. Ann. Entomol. Soc. Am., 103:956-964.

GASCON, C., WILLIAMSON, G.B. \& DA FONSECA, G.A.B. 2000. Receding forest edges and vanishing reserves. Science, 288:1356-1358.

GASTON, K.J. 2000. Global patterns in biodiversity. Nature, 405:220-227. 
GRIMBACHER, P., CATTERALL, C.P. \& KITCHING, R.L. 2008. Detecting the effects of environmental change above the species level with beetles in a fragmented tropical rainforest landscape. Ecol. Entomol., 33:66-79.

HARSTHORN, G.S. 1991. Plantas. Introducción. En: Janzen, D.H. (ed.). Historia Natural de Costa Rica. Editorial de la Universidad de Costa Rica, San José, Costa Rica. pp. 119-160

HARTSHORN, G.S. 2002. Biogeografía de los bosques neotropicales. En: Guariguata, MR and Kattan, GH (eds). Ecología y conservación de Bosques Neotropicales. Ediciones LUR. Cartago, Costa Rica. 692 pp.

HARVEY, C.A. \& SÁENZ, J.C. 2008. Evaluación y conservación de la biodiversidad en paisajes fragmentados de Mesoamérica. Editorial del Instituto Nacional de Biodiversidad, INBio. Santo Domingo de Heredia, Costa Rica. 624 pp.

HAWKINS, B.A., PORTER, E.E. \& DINIZ-FILHO, J.A.F. 2003. Productivity and history as predictors of the latitudinal diversity gradient of terrestrial birds Ecology, 84:1608-1623.

KATTAN, H.G. 2002. Fragmentación: patrones y mecanismos de extinción de especies. En: Guariguata, MR and Kattan, GH (eds). Ecología y conservación de Bosques Neotropicales. Ediciones LUR. Cartago, Costa Rica. pp. $562-590$.

KESSLER, M. 2009. The impact of population processes on patterns of species richness: Lessons from elevational gradients. Basic Appl. Ecol., 10:295-299.

KUMAR, A., LONGINO, J.T., COLWELL, R.K. \& O'DONNELL, S. 2009. Elevational patterns of diversity and abundance of eusocial paper wasps (Vespidae) in Costa Rica. Biotropica, 41:338-346.

LOMOLINO, M.V. 2001. Elevation gradients of speciesdensity: historical and prospective views. Global Ecol. Biogeogr., 10:3-13.

LONGINO, J.T. 1994. How to measure arthropod diversity in a tropical rainforest. Biol. Int., 28:3-13.

MCCAIN, C.M. 2007. Could temperature and water availability drive elevational species richness patterns? A global case study for bats. Global Ecol. Biogeogr., 16:1-13.
C.G., DA FONSECA, G.A.B. \& KENT, J. 2000. Biodiversity hotspots for conservation priorities. Nature, 403:853-858.

NICHOLS, E., LARSEN, T., SPECTOR, S., DAVIS, A.L., ESCOBAR, F., FAVILA, M. \& VULINEC, K. 2007. Global dung beetle response to tropical forest modification and fragmentation: A quantitative literature review and meta-analysis. Biol. Conserv., 137:1-19.

NOVOTNY, V. \& WEIBLEN, G.D. 2005. From communities to continents: beta-diversity of herbivorous insects. Ann. Zoologici Fennici, 42:463-475.

OBANDO, V. 2002. Biodiversidad de Costa Rica. Estado del conocimiento y gestión. Instituto Nacional de Biodiversidad. Santo Domingo de Heredia, Costa Rica. 81 pp.

ØDEGAARD, F. 2006. Host specificity, alpha- and betadiversity of phytophagous beetles in two tropical forests in Panama. Biodivers. Conserv., 15:83-105.

REID, W., MOONEY, H.A., CROPPER, A., CAPISTRANO, D., CARPENTER, S.R., CHOPRA, K., DASGUPTA, P., DIETZ, T., DURAIAPPAH KUMAR, A., HASSAN, R., KASPERSON, R., LEEMANS, R., MAY, R.M., MCMICHAEL, T.A.J., PINGALI, P., SAMPER, C., SCHOLES, R., WATSON, R.T., ZAKRI, A.H., SHIDONG, Z., ASH, N.J., BENNETT, E., KUMAR, P., LEE, M.J., RAUDSEPP-HEARNE, C., SIMONS, H., THONELL, J. \& ZUREK, M.B. 2005. Millenium ecosystem assessment synthesis. United Nations. pp. 1-219.

RICKLEFS, R.E. 2004. A comprehensive framework for global patterns in biodiversity. Ecol. Let., 7:1-15.

ROOT, T.L., PRICE, J.T., HALL, K.R., SCHNEIDER, S.H., ROSENZWEIG, C. \& POUNDS, J.A. 2003. Fingerprints of global warming on wild animals and plants. Nature, 421:57-60.

SVENNING, J.-C., ENGELBRECHT, B.M.J., KINNER, D.A., KURSAR, T.A., STALLARD, R.F. \& WRIGHT, S.J. 2006. The relative roles of environment, history and local dispersal in controlling the distributions of common tree and shrub species in a tropical forest landscape, Panama. J. Trop. Ecol., 22:575-586.

THOMAS, C.D., CAMERON, A., GREEN, R.E., BAKKE-

MYERS, N., MITTERMEIER, R.A., MITTERMEIER, 
NES, M., BEAUMONT, L.J., COLLINGHAM, Y.C., ERASMUS, B.F.N., DE SIQUEIRA, M.F., GRAINGER, A., HANNAH, L., HUGHES, L., HUNTLEY, B., VAN JAARSVELD, A.S., MIDGLEY, G.F., MILES, L., ORTEGA-HUERTA, M.A., TOWNSEND PETERSON, A., PHILLIPS, O.L. \& WILLIAMS, S.E. 2004. Extinction risk from climate change. Nature, 427:145-148.

THOMAS, S.C. \& BALTZER, J.L. 2002. Tropical forests. En: J. Wiley \& Sons (eds.). Encyclopedia of Life Sciences. Ltd: Chichester.
VALERIO, C.E. 1999. Costa Rica. Ambiente y Sociedad. Instituto Nacional de Biodiversidad, Santo Domingo de Heredia, Costa Rica. 139 pp.

VON HUMBOLDT, A. 1849. Aspects of nature in different lands and different climates, with scientific elucidations. Longman, Brown, Green \& Longman (eds.). London. U.K.

WILLIG, M.R., KAUFMAN, D.M. \& STEVENS, R.D. 2003. Latitudinal gradients of biodiversity: pattern, process, scale and synthesis. Annual Rev. Ecol. Evol. S. 34:273-309. 